\title{
Eating psychopathology and psychosocial impairment in patients treated at a Singapore eating disorders treatment programme
}

\author{
Kah Wee $\underline{\mathrm{Ng}}{ }^{1}$, MBBS, MMed, Angeline $\underline{K u e k^{2}}$, MPH, MHA, Huei Yen $\underline{L e}^{1}$, MBBCh, MMed
}

INTRODUCTION There is limited data on the psychopathology of eating disorders in Singapore. This study: (a) described levels of eating psychopathology and psychosocial impairment among individuals diagnosed with eating disorders at our hospital; and (b) compared the related psychopathology of these patients.

METHODS Between 1 August 2010 and 31 July 2012, 257 individuals who met the diagnostic criteria for eating disorders completed the Eating Disorder Examination Questionnaire (EDE-Q) and Clinical Impairment Assessment questionnaire $(\mathrm{ClA})$.

RESULTS A majority of participants were women and of Chinese ethnicity. Diagnoses included anorexia nervosa (AN; 41.6\%), bulimia nervosa (BN; 29.6\%) and eating disorder not otherwise specified (EDNOS; 28.8\%). Mean age at presentation was $20.52 \pm 7.14$ years and mean body mass index was $17.84 \pm 4.18 \mathrm{~kg} / \mathrm{m}^{2}$. Individuals with AN were significantly younger at presentation and had shorter duration of untreated illness compared to those with BN and EDNOS. There were no significant differences in the CIA scores of the diagnostic groups. Participants with BN scored higher in all subscales of the EDE-Q than those with AN and EDNOS. Our sample scored lower in most subscales of EDE-Q when compared to treatment centres in Sweden, Australia and the United States.

CONCLUSION Our clinical sample reported lower scores of psychopathology compared to overseas centres. This could be attributed to the higher percentages of BN and EDNOS diagnosed in overseas populations. Individuals with AN showed higher levels of psychopathology in our study compared to patients from the United States.

Keywords: eating disorders, psychopathology, psychosocial impairment, Singapore

\section{INTRODUCTION}

A recent epidemiological study in the United States based on the fifth edition of the Diagnostic and Statistical Manual of Mental Disorders reported that the lifetime prevalence of anorexia nervosa (AN) among women might be as high as $4 \%$ and that of bulimia nervosa (BN) to be around $2 \%$. ${ }^{(1)}$ This is a large increase when compared to another study done nearly a decade ago that reported prevalence rates of $0.3 \%-1 \%$ across diagnoses. ${ }^{(2)}$ Such trends toward thinness or features of AN were once associated with high socioeconomic status among young female North European Caucasians. ${ }^{(3)}$ The disorder and its associated behaviours and cognitions were once thought to be rare outside of Western countries, but appear to have now become more prevalent in various Asian countries, such as Taiwan, ${ }^{(4)}$ Hong Kong(5) and Japan. ${ }^{(6)}$ Similarly, the number of individuals in Singapore diagnosed with eating disorders has been on the rise over the years ${ }^{(7-9)}$ - from six new presentations in 1994 to 24 in $2002 ; ;^{(7)}$ a decade later in 2012, the numbers have increased to 163 newly diagnosed individuals ${ }^{(9)}$ at the Singapore General Hospital (SGH), Singapore.

Eating disorder psychopathology can be experienced in various domains, such as restricting one's diet, concerns over one's body size and shape, and fear of weight gain. Such domains can be measured using the Eating Disorder Examination Questionnaire (EDE-Q). A study reported that preference for thinness is the ideal among young Chinese Singapore women, even though they might be underweight. ${ }^{(10)}$ This echoes the findings of a large sample study that investigated the degree of body dissatisfaction among Chinese undergraduates in Hong Kong. ${ }^{(5)}$ Another study that examined the restraint and eating concerns of young women in Australia and Singapore revealed that Singaporean Chinese women in the control group had greater overall eating disorder psychopathology compared to other cultural groups and greater restraint than North European Australians or expatriates. ${ }^{(11)}$ However, a study from the United States that investigated the profile and clinical features of adolescents who underwent family therapy trial for AN reported that Asian American adolescents scored lower in the EDE-Q than their non-Asian peers, significantly in the restraint and weight concern subscales. ${ }^{(12)}$

A study that compared the psychopathology of young women of different ethnicities in Australia and Singapore reported that those with eating disorders had similar psychopathology across ethnicities, whereas a higher level of eating disorder psychopathology was found in Singaporean controls than their Australian counterparts and expatriate Singaporeans. ${ }^{(11,13)}$ Another study examined the level of eating disorder behaviour in healthy controls from Australia and Singapore using the EDE-Q. ${ }^{(14)}$ It reported that even though the overall levels of eating disorder psychopathology were very similar between the two populations, Singaporean women were found to be more

${ }^{1}$ Department of Psychiatry, Singapore General Hospital, Singapore, ${ }^{2}$ Australasian College of Health Service Management, Victoria, Australia

Correspondence: Dr Ng Kah Wee, Consultant, Department of Psychiatry, Singapore General Hospital, 20 College Road, Academia, Singapore 169865. ng.kah.wee@singhealth.com.sg 
fearful of losing control over their eating and of weight gain or becoming fat, and more anxious at the prospect of weighing themselves regularly. Such findings seem to suggest that levels of eating psychopathology may be high in the general Singapore population.

The Eating Disorder Examination Interview $(E D E)^{(15)}$ is considered the gold standard for assessing eating disorder psychopathology, but is more time-consuming and costly, as a trained assessor is required to administer the semi-structured interview. The adapted EDE-Q, however, has good psychometric properties $^{(16)}$ and can be used as a clinical measure of treatment response. Being a self-reported instrument, a possible advantage of the EDE-Q is that the respondent may be more willing to disclose certain sensitive behaviours deemed to be shameful and secretive. ${ }^{(17)}$ On the one hand, eating disorders are associated with negative impact on psychosocial functioning and lower quality of life; ${ }^{(18)}$ on the other, this psychosocial impairment leads one to seek help and treatment. ${ }^{(19)}$ The Clinical Impairment Assessment questionnaire $(\mathrm{CIA})$ is a tool designed to specifically assess the severity of psychosocial impairment due to an eating disorder and is to be filled in by the respondent immediately after completing a measure of current eating disorder symptoms. ${ }^{(20)}$

We aimed to use the above instruments to determine: (a) the level of eating psychopathology and psychosocial impairment among individuals diagnosed with an eating disorder at our hospital; and (b) if there were any differences in levels of eating psychopathology and psychosocial impairment among individuals with different eating disorder classifications. We hypothesised that the level of eating psychopathology and psychosocial impairment of our clinical population would be similar to that in overseas treatment centres. We also hypothesised that participants with $\mathrm{BN}$ would experience higher levels of psychopathology when compared to those with $\mathrm{AN}$.

\section{METHODS}

Participants were recruited from our hospital's eating disorder treatment programme for patients aged $\geq 12$ years. Referrals were from multiple sources, including inpatient wards, clinics, other hospitals, community clinics and self-referrals.

Individuals who saw a psychiatrist at SGH for the first time between 1 August 2010 and 31 July 2012 were asked to complete a set of self-reported questionnaires, consisting of a demographical data sheet, the EDE-Q and CIA. The study was approved by the institutional research review board. Written consent was obtained from participants aged $\geq 21$ years, while for participants who were minors, consent was obtained from both the individual and parent. Eating disorder diagnoses were made by the treating psychiatrist according to the Diagnostic and Statistical Manual of Mental Disorders, fourth edition. Participants were included if they were diagnosed with $\mathrm{AN}, \mathrm{BN}$ or eating disorder not otherwise specified (EDNOS) and had completed both questionnaires. Within the study's time frame, 257 individuals met the inclusion criteria. Individuals were excluded from the study if they were not diagnosed with an eating disorder, had not completed the questionnaires or did not provide consent.
The EDE-Q version 6.0 is a self-reported, adapted version of the widely used EDE. It focuses on the past 28 days and scores on a 7-point Likert scale. It comprises four subscales designed to measure the domains of dietary restraint, weight concern, shape concern and eating concern. ${ }^{(17)}$ An average of the subscale score is calculated when at least half of the items in each subscale have been completed. Higher scores indicate higher levels of psychopathology severity or frequency. A global score is obtained from the average of four subscales and has been shown to have high internal consistency (Cronbach's alpha 0.93). ${ }^{(21)}$ It must be noted, however, that EDE-Q validity studies have not been done with a Singaporean population.

The CIA is a 16-item self-reported questionnaire that measures the severity of psychosocial impairment due to features of eating disorders $^{(20)}$ in the past 28 days. It is designed to be administered immediately after the EDE-Q or an equivalent eating disorder psychopathology measurement tool. Each item is scored against a 4-point Likert scale and the scores are calculated to generate a single global score, with the requirement that at least 12 items have to be completed. The CIA has been shown to have good internal consistency (Cronbach's alpha 0.97) and acceptable overall test-retest reliability, with an intraclass correlation coefficient of $0.86 .{ }^{(19)}$ The best cutoff point was seen to be a global CIA score of 16 , with $76 \%$ sensitivity and $86 \%$ specificity. ${ }^{(19)}$ It was also reported that the global CIA score can predict case status, ${ }^{(19)}$ with a higher score being indicative of a higher level of psychosocial impairment. However, ClA validity studies have also not been conducted on a Singaporean population.

SPSS Statistics version 17.0 for Windows XP (SPSS Inc, Chicago, IL, USA) was used for data analysis. Categorical data was presented as frequencies and percentages, and Pearson's chisquare test was used to analyse demographic differences between types of eating disorders. For continuous variables, differences between the various eating disorder diagnoses were compared using one-way analysis of variance and Tukey post-hoc tests, with statistical significance set at $p<0.05$.

\section{RESULTS}

A majority of participants were women and of Chinese ethnicity (Table I). Other ethnicities (14.0\%) included Bangladeshi, Caucasian, Chilean, Eurasian, Filipino, Korean, Mongolian and Vietnamese. As individuals were referred from countries other than Singapore, the varied ethnicities and nationalities in our group were according to expectation.

Eating disorder diagnoses among our participants included AN (41.6\%), BN (29.6\%) and EDNOS (28.8\%). About half of our participants had at least one psychiatric comorbidity, with the top three conditions being major depressive disorder (63.8\%), obsessive compulsive disorder (5.4\%) and some form of anxiety disorder (4.6\%). Other comorbid psychiatric conditions included bipolar disorder, personality disorder, adjustment disorder, substance abuse, alcohol dependence, conduct disorder, gender identity disorder, post-traumatic stress disorder, panic disorder, attention deficit and hyperactivity disorder, and the presence of depressive and/or anxiety traits. About a third (32.7\%) of our 
Table I. Demographics of individuals diagnosed with eating disorders $(n=257)$.

\begin{tabular}{|c|c|c|c|c|c|c|}
\hline \multirow[t]{2}{*}{ Variable } & \multicolumn{4}{|c|}{ No. (\%) } & \multirow[t]{2}{*}{$\chi^{2}$} & \multirow[t]{2}{*}{ p-value } \\
\hline & Total & AN $(n=107)$ & BN $(n=76)$ & EDNOS $(n=74)$ & & \\
\hline Gender & & & & & 0.65 & 0.723 \\
\hline Women & $238(92.6)$ & $100(93.5)$ & $71(93.4)$ & $67(90.5)$ & & \\
\hline Men & $19(7.4)$ & $7(6.5)$ & $5(6.6)$ & $7(9.5)$ & & \\
\hline Ethnicity & & & & & 4.42 & 0.62 \\
\hline Indian & $14(5.4)$ & $7(6.5)$ & $3(3.9)$ & $4(5.4)$ & & \\
\hline Malay & $9(3.5)$ & $1(0.9)$ & $4(5.3)$ & $4(5.4)$ & & \\
\hline Other* & $32(12.5)$ & $12(11.2)$ & $10(13.2)$ & $10(13.5)$ & & \\
\hline Psychiatric comorbidity & & & & & 0.95 & 0.623 \\
\hline Yes & $130(50.6)$ & $52(48.6)$ & $42(55.3)$ & $36(48.6)$ & & \\
\hline Suicide attempt/self-harm behaviour & & & & & 10.53 & $0.005^{+}$ \\
\hline Yes & $84(32.7)$ & $23(21.5)$ & $30(39.5)$ & $31(41.9)$ & & \\
\hline No & $173(67.3)$ & $84(78.5)$ & $46(60.5)$ & $43(58.1)$ & & \\
\hline
\end{tabular}

*Including participants of Bangladeshi, Caucasian, Chilean, Eurasian, Filipino, Korean, Mongolian and Vietnamese ethnicities. $+p<0.05$ was statistically significant. AN: anorexia nervosa; BN: bulimia nervosa; EDNOS: eating disorder not otherwise specified

participants had a history of suicide attempts and/or self-harm. Participants with the diagnoses of $\mathrm{BN}$ and EDNOS were more likely to have a history of suicide attempt or self-harm behaviours compared to those diagnosed with $\mathrm{AN}(\mathrm{p}=0.005)$. However, there were no statistically significant differences between the diagnostic groups in terms of ethnicity and comorbid psychiatric conditions. Participants with BN were more likely to have a comorbid psychiatric condition when compared to participants with other eating disorder diagnoses, although the difference did not reach significance. Some $(n=83,32.3 \%)$ participants had a medical complication related to eating disorder, and approximately half of these participants were diagnosed with AN. The top three medical complications were bradycardia $(n=34)$, low bone density for chronological age $(n=18)$ and gastritis ( $n=15)$. However, there was no statistically significant difference between the diagnostic groups for the occurrence of medical complications ( $p=0.158)$.

The mean age at presentation of our study cohort was $20.52 \pm 7.14$ years (Table II). When compared to participants with $\mathrm{BN}$ and EDNOS, those with $\mathrm{AN}$ presented at a younger age and the difference was significant $\left(F_{(2,254)}=5.437 ; \mathrm{p}=0.005\right)$. A Tukey post-hoc test found that participants with AN presented at a significantly younger age (18.82 \pm 6.02 years) compared to those with $\mathrm{BN}(21.53 \pm 5.88$ years; $\mathrm{p}=0.029)$ and EDNOS $(21.95 \pm 9.13$ years; $p=0.010)$ (Table II). However, there was no statistically significant difference in age at presentation between the $\mathrm{BN}$ and EDNOS groups. The mean duration of untreated illness (DUI) was $3.63 \pm 5.25$ years. DUI was shorter in participants with AN when compared to those with BN and EDNOS, with the difference being statistically significant $\left(F_{(2,254)}=6.821 ; \mathrm{p}=0.001\right)$. A Tukey post-hoc test revealed that participants with AN had a shorter DUI $(2.24 \pm 2.94$ years $)$ when compared to those with $\mathrm{BN}(4.82 \pm 4.78$ years; $\mathrm{p}=0.003)$ and EDNOS (4.42 \pm 7.43 years; $p=0.015)$, although the difference between the $\mathrm{BN}$ and EDNOS groups was not significant (Table II).
The mean body mass index (BMI) was $17.84 \pm 4.18 \mathrm{~kg} / \mathrm{m}^{2}$ and participants with AN presented with a significantly lower BMI $\left(14.71 \pm 1.65 \mathrm{~kg} / \mathrm{m}^{2}\right)$ than those with $\mathrm{BN}$ and EDNOS $\left(F_{(2,254)}=109.236 ; \mathrm{p}<0.001\right)$, as expected. A Tukey post-hoc test also found that participants with EDNOS $\left(18.70 \pm 3.72 \mathrm{~kg} / \mathrm{m}^{2}\right)$ presented with a significantly lower BMI when compared to those with BN $\left(21.41 \pm 3.85 \mathrm{~kg} / \mathrm{m}^{2} ; \mathrm{p}<0.001\right)$ (Table II). Overall, participants had a mean inpatient stay of $4.53 \pm 3.63$ weeks and no significant difference was found between the different groups.

The mean CIA and EDE-Q scores of participants are presented in Table III. The overall mean CIA score was 26.67 \pm 13.00 , and although the BN group had a higher mean CIA score $(29.12 \pm 12.02)$ than the AN $(25.93 \pm 13.75)$ and EDNOS $(25.22 \pm 12.66)$ groups, the difference in the CIA scores between the various groups was not significant. When EDE-Q subscale scores were compared, the BN group had a significantly higher restraint score when compared to the $\mathrm{AN}$ and EDNOS groups $\left(F_{(2,254)}=4.569 ; \mathrm{p}=0.011\right)$. A Tukey post-hoc test found that the $\mathrm{BN}$ group had a significantly higher restraint score $(3.61 \pm$ 1.55) compared to the AN $(2.94 \pm 1.94 ; \mathrm{p}=0.043)$ and EDNOS $(2.76 \pm 1.95 ; p=0.014)$ groups (Table III). However, no significant differences were seen in the mean restraint scores between the $\mathrm{AN}$ and EDNOS groups. The BN group also had a higher eating concern score when compared to the AN and EDNOS groups, and this difference was significant $\left(F_{(2,254)}=4.793 ; p=0.009\right)$. A Tukey post-hoc test also found that the $\mathrm{BN}$ group had a significantly higher eating concern score $(3.56 \pm 1.35)$ when compared to the $\operatorname{AN}(2.88 \pm 1.78, p=0.015)$ and EDNOS $(2.87 \pm 1.84, p=0.025)$ groups, but there was no significant difference in the mean eating concern scores between the AN and EDNOS groups (Table III).

Similarly, when the shape concern scores were compared, although no significant differences were seen between the AN and EDNOS groups, the $\mathrm{BN}$ group had a significantly higher shape concern score when compared to the other groups $\left(F_{(2,254)}=8.544\right.$; $\mathrm{p}<0.001)$. A Tukey post-hoc test showed that the BN group had 
Table II. Characteristics of individuals diagnosed with eating disorders.

\begin{tabular}{|c|c|c|c|c|c|c|c|}
\hline \multirow[t]{2}{*}{ Variable } & \multicolumn{4}{|c|}{ Mean \pm standard deviation } & \multirow[t]{2}{*}{$\boldsymbol{F}$} & \multirow[t]{2}{*}{ df } & \multirow[t]{2}{*}{ p-value } \\
\hline & Total & AN $(n=107)$ & $\mathrm{BN}(n=76)$ & EDNOS $(n=74)$ & & & \\
\hline Age at presentation (yr) & $20.52 \pm 7.14$ & $18.82 \pm 6.02$ & $21.53 \pm 5.88$ & $21.95 \pm 9.13$ & 5.437 & 2,254 & $0.005^{*}$ \\
\hline Age at onset (yr) & $16.79 \pm 5.51$ & $16.34 \pm 4.36$ & $16.71 \pm 4.48$ & $17.53 \pm 7.57$ & 1.024 & 2,253 & 0.361 \\
\hline Duration of untreated illness (yr) & $3.63 \pm 5.25$ & $2.24 \pm 2.94$ & $4.82 \pm 4.78$ & $4.42 \pm 7.43$ & 6.821 & 2,253 & $0.001^{*}$ \\
\hline Weight at presentation (kg) & $46.23 \pm 12.13$ & $37.44 \pm 5.20$ & $56.14 \pm 10.24$ & $48.77 \pm 12.12$ & 95.858 & 2,254 & $<0.001^{*}$ \\
\hline BMI at presentation $\left(\mathrm{kg} / \mathrm{m}^{2}\right)$ & $17.84 \pm 4.18$ & $14.71 \pm 1.65$ & $21.41 \pm 3.85$ & $18.70 \pm 3.72$ & 109.236 & 2,254 & $<0.001^{*}$ \\
\hline Duration of inpatient admission (wk) & $4.53 \pm 3.63$ & $4.60 \pm 2.95$ & $3.63 \pm 2.56$ & $4.76 \pm 5.44$ & 0.286 & 2,67 & 0.752 \\
\hline
\end{tabular}

${ }^{*} p<0.05$ was statistically significant. AN: anorexia nervosa; BMI: body mass index; BN: bulimia nervosa; df: degrees of freedom; EDNOS: eating disorder not otherwise specified

Table III. CIA and EDE-Q scores of individuals diagnosed with eating disorders $(\mathrm{n}=\mathbf{2 5 7}$ ).

\begin{tabular}{|c|c|c|c|c|c|c|}
\hline \multirow[t]{2}{*}{ Variable } & \multicolumn{4}{|c|}{ Mean \pm standard deviation } & \multirow[t]{2}{*}{$\boldsymbol{F}$} & \multirow[t]{2}{*}{ p-value } \\
\hline & Total & AN $(n=107)$ & $\mathrm{BN}(n=76)$ & EDNOS $(n=74)$ & & \\
\hline CIA score & $26.67 \pm 13.00$ & $25.93 \pm 13.75$ & $29.12 \pm 12.02$ & $25.22 \pm 12.66$ & 2.003 & 0.137 \\
\hline \multicolumn{7}{|l|}{ EDE-Q score } \\
\hline Restraint & $3.09 \pm 1.86$ & $2.94 \pm 1.94$ & $3.61 \pm 1.55$ & $2.76 \pm 1.95$ & 4.569 & $0.011^{*}$ \\
\hline Eating concern & $3.09 \pm 0.17$ & $2.88 \pm 1.78$ & $3.56 \pm 1.35$ & $2.87 \pm 1.84$ & 4.793 & $0.009 *$ \\
\hline Weight concern & $3.38 \pm 1.77$ & $2.97 \pm 1.77$ & $4.03 \pm 1.33$ & $3.30 \pm 1.99$ & 8.533 & $<0.001^{*}$ \\
\hline Global score & $3.33 \pm 1.59$ & $3.07 \pm 1.62$ & $3.91 \pm 1.15$ & $3.11 \pm 1.79$ & 7.547 & $<0.001^{*}$ \\
\hline
\end{tabular}

${ }^{*} \mathrm{p}<0.05$ was statistically significant. AN: anorexia nervosa; BN: bulimia nervosa; CIA: Clinical Impairment Assessment questionnaire; EDE-Q: Eating Disorder Examination Questionnaire; EDNOS: eating disorder not otherwise specified

a significantly higher shape concern score of $4.41 \pm 1.19$ when compared to the AN $(3.42 \pm 1.74 ; \mathrm{p}<0.001)$ and EDNOS $(3.54$ $\pm 1.98 ; \mathrm{p}=0.005$ ) groups (Table III). Likewise, the BN group had a higher weight concern score when compared to the AN and EDNOS groups $\left(F_{(2,254)}=8.533 ; \mathrm{p}<0.001\right)$, with a post-hoc Tukey test revealing that compared to the AN $(2.97 \pm 1.77 ; \mathrm{p}<0.001)$ and EDNOS (3.30 $\pm 1.99 ; \mathrm{p}=0.027)$ groups, the $\mathrm{BN}$ group had a weight concern score of $4.03 \pm 1.33$ (Table III). However, again, no significant differences were seen in the mean weight concern scores between the AN and EDNOS groups.

These findings suggest that participants with $\mathrm{BN}$ experienced a higher intensity of eating disorder psychopathology in all domains of EDE-Q when compared to those with AN and EDNOS. Thus, it is not surprising that the BN group's mean EDE-Q global score was also significantly higher than that of the AN and EDNOS groups $\left(F_{(2,254)}=7.547 ; \mathrm{p}<0.001\right)$. As earlier, post-hoc Tukey tests revealed that the $\mathrm{BN}$ group had a higher EDE-Q global score $(3.91 \pm 1.15)$ when compared to the AN $(3.07 \pm 1.62 ; p=0.001)$ and EDNOS ( $3.11 \pm 1.79 ; p=0.005)$ groups, but no significant difference was seen in the mean EDE-Q global scores between the AN and EDNOS groups (Table III).

\section{DISCUSSION}

As of June 2014, the Singapore population was 5.47 million, and its ethnic profile was $76.2 \%$ Chinese, $15.0 \%$ Malay, $7.4 \%$ Indian and $1.4 \%$ others. ${ }^{(22)}$ The proportion of Chinese participants $(78.6 \%)$ in our sample appeared to reflect the demographic distribution of the general Singapore population. However, this was not so for participants of other ethnicities - the Malay ethnicity was under-represented (study sample 3.5\% vs. Singapore population $15.0 \%$ ) while other ethnicities were over-represented (study sample $12.5 \%$ vs. Singapore population $1.4 \%$ ). The overrepresentation of individuals of other ethnicities in our clinical sample was expected, as the treatment programme at our hospital receives referrals from overseas centres. Among participants categorised as 'others' in our study, nearly half were Caucasian. Another possible explanation for the over-representation of other ethnicities may be the lower stigma associated with consulting psychiatrists in Western culture, and thus, more individuals of Caucasian ethnicity may be willing to seek help at our centre. ${ }^{(23)}$

Similar to previous studies, ${ }^{(24)}$ we found consistent underrepresentation of Malay and Indian ethnicities in our sample. In a study of 50 individuals with eating disorders treated at another general hospital in Singapore between 1991 and 1996, the ethnic make-up of patients of Malay and Indian ethnicities was both low at $10 \% .{ }^{(25)}$ These findings were not in agreement with an earlier study of over 4,000 Singaporean women, in which $20.6 \%$ of Malay women and $9.4 \%$ of Indian women were found to be at risk of developing eating disorder. ${ }^{(26)}$ It was previously suggested that the Malay and Indian cultures may be protective factors against the development of eating disorder due to lower emphasis placed on the thin ideal therein. ${ }^{(7)}$ The under-representation of Malay ethnicity in our sample could also be explained by the lower likelihood of Malay individuals seeking help for psychiatric illnesses. ${ }^{(27,28)}$ To our knowledge, there is currently no literature on the help-seeking behaviour of the Indian community in Singapore. However, it was reported that Indian women in the United Kingdom were less likely to 
seek help for psychiatric illnesses compared to their Caucasian counterparts ${ }^{(29)}$ because of the lack of knowledge about associated conditions and the belief that treatment would not be beneficial. We may extrapolate such theories to the Indian community in Singapore. Poor eating disorder literacy in Singapore could also be a compounding factor. ${ }^{(30)}$ Future studies on the characteristics of eating disorders and cultural factors that influence eating disorders among individuals of Malay and Indian ethnicities would be of value.

Our study reported a significantly shorter DUI among participants with AN compared to those from the other diagnostic groups. Against the background of poor eating disorder literacy in Singapore, ${ }^{(30)}$ it is likely that individuals with AN may have presented to the clinic only because of telling signs such as severe weight loss and physical manifestations of medical complications, while those with BN or EDNOS might have sought help only after a longer duration. Participants with $\mathrm{AN}$ in our study presented at a significantly younger age compared to those with $\mathrm{BN}$ and EDNOS (AN $18.82 \pm 6.02$ years, BN $21.53 \pm 5.88$ years, EDNOS $21.95 \pm 9.13$ years; $p=0.005$ ), in keeping with studies from the United Kingdom. ${ }^{(31,32)}$

Participants with BN and EDNOS in our study had higher rates of suicide attempts and self-harm behaviour when compared to those with $\mathrm{AN}(\mathrm{p}=0.005)$. The $\mathrm{BN}$ diagnostic group also had higher rates of comorbid psychiatric conditions, although this did not reach statistical significance $(p=0.623)$. Our findings mirror studies from the United States - one study reported elevated rates of self-harm and substance use among patients with $\mathrm{BN}$ and binge eating disorder, ${ }^{(33)}$ while another concluded that BN patients with a family history of drug abuse were more likely to have been treated for drug abuse problems themselves. ${ }^{(34)}$ The higher rates of comorbid psychiatric conditions and self-harm behaviour among individuals with BN and EDNOS in our sample could be extrapolated to explain the need for hospitalisation, even though treatment for such patients is usually outpatient-focused.

In our study, the longer DUI among individuals with BN could explain the higher levels of eating disorder psychopathology seen among these individuals when compared to those with AN and EDNOS. Participants with AN were also closer to the adolescent age group when compared to those with $\mathrm{BN}$ and EDNOS. This finding echoes a previous study in which adolescents with AN tended to report lower scores of eating psychopathology, as symptoms tended to be ego-syntonic and were not recognised as pathological. ${ }^{(35)}$ Although the $\mathrm{BN}$ group scored highest in all domains of the EDE-Q with a high corresponding CIA score, the latter did not reach statistical significance, suggesting that higher EDE-Q scores did not necessarily translate to higher levels of psychosocial impairment. This could be attributed to individuals with AN presenting with very low body weights that required longer periods of hospitalisation when compared to those with $\mathrm{BN}$, possibly contributing to a degree of psychosocial impairment.

The EDE-Q subscale scores in our study were similar to those found in earlier studies, ${ }^{(11,13)}$ which may imply that there was a consistent trend from 2007 to 2012 for body image concerns and eating psychopathology in our local population.
The profile of participants in our study was also similar to the Singapore arm of studies by Soh et $\mathrm{al}^{(11,13)}$ in terms of gender and ethnicity distributions, and average age and BMI at presentation, suggesting that the demographic characteristics of new presentations have not shifted much over the years. Although these studies had a small sample size of Singaporean Chinese patients, they were the only local studies with which we could compare our cohort.

Eating disorders were thought to be an illness associated with people of North European Caucasian ethnicity and considered relatively rare outside of Western countries. However, examining the eating disorders in our local population allows us to compare and contrast the underlying psychopathology with that of overseas centres. Comparisons with other studies of similar design suggest that our participants scored lower than their overseas counterparts ${ }^{(11,36-39)}$ in most EDE-Q subscales. Compared to a study from the United Kingdom, ${ }^{(37)}$ which reported an EDE-Q restraint subscale score of $3.57( \pm 1.41)$ and EDE-Q weight concern subscale score of $2.67( \pm 1.44)$, our participants scored lower in the respective subscales. Similarly, when compared to a study from Sweden, ${ }^{(38)}$ which reported EDE-Q scores of $3.67( \pm 1.60)$ for restraint, $3.36( \pm 1.34)$ for eating concern, $4.72( \pm 1.32)$ for shape concern, 4.07 ( \pm 1.39$)$ for weight concern and $4.06( \pm 1.20)$ for global score, our participants scored lower in all subscales. In comparison with an Australian study ${ }^{(11)}$ that reported 3.2 for restraint, 4.5 for shape concern, 3.9 for weight concern and 3.8 for global score, our cohort reported lower restraint, shape concern, weight concern and global EDE-Q scores but had similar CIA scores. ${ }^{(39)}$ These differences in scores may be explained by the higher proportion of patients with AN having low body weight in our sample, while the other quoted studies included older participants who had BN and EDNOS as major diagnoses. ${ }^{(36-39)}$

We compared our scores differentially with one American study ${ }^{(40)}$ that reported the EDE-Q scores for patients with AN and $\mathrm{BN}$. Their study comprised a sample of AN patients with mean BMI of $16.49 \mathrm{~kg} / \mathrm{m}^{2}$ who scored 1.7 ( \pm 1.98$)$ for restraint, 1.39 $( \pm 1.43)$ for eating concern, $1.88( \pm 1.84)$ for weight concern and $2.21( \pm 1.95)$ for shape concern subscales on the EDE-Q. Their sample of $\mathrm{BN}$ patients reported $4.50( \pm 1.16)$ for restraint, 4.40 $( \pm 1.16)$ for eating concern, 4.81 ( \pm 1.39) for weight concern and 4.95 ( \pm 1.19$)$ for shape concern. In comparison, individuals who had AN in our study scored higher in all EDE-Q subscales, while those with $\mathrm{BN}$ scored lower in all subscales. Individuals with AN also presented with lower mean BMI. Indeed, EDE-Q scores and perceived societal norms for eating behaviours and body image can vary between countries and cultures. ${ }^{(38)}$ From our comparisons, we can perhaps extrapolate that when compared to their overseas counterparts, individuals with AN in Singapore experienced higher levels of eating disorder psychopathology, whereas those with BN did not. Further evaluation of other studies with EDE-Q and CIA scores specific to each diagnostic group is needed to lend more weight to these findings.

Our study was not without limitations. First, retrospective recall of information was part of our study design, particularly for the scoring of the EDE-Q and CIA questionnaires. Second, 
the EDE-Q and CIA questionnaires have not yet been validated for the Singapore context. Nonetheless, they remain useful for measuring the degree of eating disorder psychopathology in the Singapore clinical population. Third, our eating disorder treatment programme caters not only to adults but also underage patients. It is possible that some participants were reluctantly taken to treatment by their parents and that the severity of their symptoms was downplayed, falsely influencing the EDE-Q and $\mathrm{CIA}$ scores reported. Fourth, participants were diagnosed based on the clinical assessment of the treating psychiatrist rather than by using a validated diagnostic tool. Fifth, given that our data was collected from a single centre and there was under-representation of the Malay and Indian ethnicities when compared to the general Singapore population, the generalisability of our findings could be questioned. These shortcomings notwithstanding, our study still represents a valuable addition to the existing literature on individuals with eating disorders in Singapore.

Our findings suggested that the eating psychopathology of our local population, which appeared unchanged over time, still differed from that of a Caucasian population. Significant findings were: (a) under-representation of Malay and Indian ethnicities; (b) participants with $\mathrm{AN}$ presented earlier in the course of illness, had lower rates of self-harm behaviours, and scored lower in the EDE-Q and CIA subscales; and (c) participants with $\mathrm{BN}$ reported high scores in all domains of the EDE-Q. These findings suggest the need for an individualised approach when treating individuals with eating disorders, taking into account the possible effects of ethnicity and cultural differences. Nevertheless, ethnic and cultural practices could also be tapped into as complements that may aid in a patient's recovery. Future studies that look into how ethnicity and cultural factors impact the course of eating disorders would be valuable.

\section{REFERENCES}

1. Smink FR, van Hoeken D, Hoek HW. Epidemiology, course, and outcome of eating disorders. Curr Opin Psychiatry 2013; 26:543-8.

2. Hoek HW, van Hoeken D. Review of the prevalence and incidence of eating disorders. Int J Eat Disord 2003; 34:383-96.

3. Kok LP, Tian CS. Susceptibility of Singapore Chinese schoolgirls to anorexia nervosa--Part I (Psychological factors). Singapore Med J 1994; 35:481-5.

4. Yeh HW, Tzeng NS, Chu H, et al. The risk of eating disorders among female undergraduates in Taiwan. Arch Psychiatr Nurs 2009; 23:430-40.

5. Lee S, Leung T, Lee AM, Yu H, Leung CM. Body dissatisfaction among Chinese undergraduates and its implications for eating disorders in Hong Kong. Int J Eat Disord 1996; 1:77-84.

6. Nakai $Y$, Nin K, Fukushima $M$, et al. Eating disorder questionnaire (EDE-Q): norms for undergraduate Japanese women. Eur Eat Disord Rev 2014; 22:439-42.

7. Lee HY, Lee EL, Pathy P, Chan YH. Anorexia nervosa in Singapore: an eight-year retrospective study. Singapore Med J 2005; 46:275-81.

8. Ung EK. Eating disorders in Singapore: a review. Ann Acad Med Singapore 2003; 32:19-24.

9. Lee HY, Hoodbhoy Z. You are worth more than you weigh: preventing eating disorders. Ann Acad Med Singapore 2013; 42:64-5.

10. Wang MC, Ho TF, Anderson JN, Sabry ZI. Preference for thinness in Singapore--a newly industrialised society. Singapore Med J 1999; 40:502-7.

11. Soh NL, Touyz S, Dobbins TA, et al. Restraint and eating concern in North European and East Asian women with and without eating disorders in Australia and Singapore. Aust N Z J Psychiatry 2007; 41:536-45.

12. Lee HY, Lock J. Anorexia nervosa in Asian-American adolescents: do they differ from their non-Asian peers. Int J Eat Disord 2007; 40:227-31.

13. Soh NL, Touyz S, Dobbins TA, et al. Body image disturbance in young North European and East Asian women with and without eating disorders in Australia and Singapore. Eur Eat Disord Rev 2008; 16:287-96.

14. Mond JM, Chen A, Kumar R. Eating-disordered behavior in Australian and Singaporean women: a comparative study. Int J Eat Disord 2010; 43:717-23.

15. Fairburn CG, Cooper Z. The eating disorder examination. In: Fairburn CG, Wilson GT, eds. Binge eating: Nature, assessment, and treatment. 12th ed. New York: Guilford Press, 1993: 317-60.

16. Mond JM, Hay PJ, Rodgers B, Owen C, Beumont PJ. Temporal stability of the Eating Disorder Examination Questionnaire. Int J Eat Disord 2004; 36:195-203.

17. Fairburn CG, Beglin SJ. Assessment of eating disorders: interview or self-report questionnaire? Int J Eat Disord 1994; 16:363-70.

18. Mitchison D, Hay P, Mond J, Slewa-Younan S. Self-reported history of anorexia nervosa and current quality of life: findings from a community-based study. Qual Life Res 2012; 22:273-81.

19. Bohn K, Doll HA, Cooper Z, et al. The measurement of impairment due to eating disorder psychopathology. Behav Res Ther 2008; 46:1105-10.

20. Bohn K, Fairburn CG. Clinical Impairment Assessment Questionnaire (CIA 3.0). In: Fairburn CG. Cognitive Behavior Therapy and Eating Disorders. New York: Guilford Press, 2008: 315-7.

21. Fairburn CG. Cognitive behavior therapy and eating disorders. New York: Guilford Press, 2008.

22. National Population and Talent Division, Singapore. 2014 Population in Brief. Available at: http://www.nptd.gov.sg/portals/0/news/population-in-brief-2014. pdf. Accessed December 9, 2015.

23. Leong FT, Lau AS. Barriers to providing effective mental health services to Asian Americans. Ment Health Serv Res 2001; 3:201-14.

24. Kuek A, Utpala R, Lee HY. The clinical profile of patients with anorexia nervosa in Singapore: a follow-up descriptive study. Singapore Med J 2015; 56:324-8.

25. Ung EJ, Lee S, Kua EH. Anorexia nervosa and bulimia--a Singapore perspective. Singapore Med J 1997; 38:332-5.

26. Ho TF, Tai BC, Lee EL, Cheng S, Liow PH. Prevalence and profile of females at risk of eating disorders in Singapore. Singapore Med J 2006; 47:499-503.

27. Peh AL, Tay LK. Demographic profile and clinical features of patients with bipolar disorder in an outpatient setting in Singapore. Singapore Med J 2008; 49:380-3.

28. Ang AW, Ko SM, Kua EH. Psychiatric referrals from an accident and emergency department in Singapore. J Accid Emerg Med 1995; 12:119-22.

29. Taylor R, Brown JS, Weinman J. A comparison of the illness perceptions of North Indian and white British women. J Ment Health 2013; 22:22-32.

30. Chen A, Mond JM, Kumar R. Eating disorders mental health literacy in Singapore: beliefs of young adult women concerning treatment and outcome of bulimia nervosa. Early Interv Psychiatry 2010; 4:39-46.

31. Brewin N, Baggott J, Dugard P, Arcelus J. Clinical normative data for eating disorder examination questionnaire and eating disorder inventory for DSM-5 feeding and eating disorder classifications: a retrospective study of patients formerly diagnosed via DSM-IV. Eur Eat Disord Rev 2014; 4:299-305.

32. Mountford V, Haase AM, Waller G. Is body checking in the eating disorders more closely related to diagnosis or to symptom presentation? Behav Res Ther 2007; 45:2704-11.

33. Dohm FA, Striegal-Moore RH, Wilfley DE, et al. Self-harm and substance use in a community sample of Black and White women with binge eating disorder or bulimia nervosa. Int J Eat Disord 2002; 32:389-400.

34. Mitchell JE, Hatsukami D, Pyle R, Eckert E. Bulimia with and without a family history of drug abuse. Addict Behav 1998; 13:245-51.

35. Passi VA, Bryson SW, Lock J. Assessment of eating disorders in adolescents with anorexia nervosa: self-report questionnaire versus interview. Int J Eat Disord 2003; 33:45-54.

36. Soh NL, Touyz SW, Surgenor LJ. Eating and body image disturbances across cultures: a review. Eur Eat Disord Rev 2006; 14:54-65.

37. Shafran R, Lee M, Cooper Z, Palmer RL, Fairburn CB. Effect of psychological treatment on attentional bias in eating disorders. Int J Eat Disord 2008; 41:348-54.

38. Welch E, Birgegård A, Parling T, Ghaderi A. Eating disorder examination questionnaire and clinical impairment assessment questionnaire: general population and clinical norms for young adult women in Sweden. Behav Res Ther $2011 ; 49: 85-91$.

39. Scott N, Hanstock TL, Thornton C. Dysfunctional self-talk associated with eating disorder severity and symptomatology. J Eat Disord 2014; 2:14.

40. Binford RB, Le Grange D, Jellar CC. Eating disorders examination versus eating disorders examination-questionnaire in adolescents with full and partial-syndrome bulimia nervosa and anorexia nervosa. Int J Eat Disord 2005; 37:44-9. 